Thomas LACROIX, Les réseaux marocains du développement - géographie du transnational et politiques du territorial

\title{
Christian Pihet
}

\section{(2) OpenEdition}

Journals

Édition électronique

URL : https://journals.openedition.org/remi/4094

DOI : 10.4000/remi.4094

ISSN : $1777-5418$

Éditeur

Université de Poitiers

\section{Édition imprimée}

Date de publication : 1 juillet 2006

Pagination : 177-179

ISBN : 2-911627-42-3

ISSN : 0765-0752

Référence électronique

Christian Pihet, "Thomas LACROIX, Les réseaux marocains du développement - géographie du transnational et politiques du territorial », Revue européenne des migrations internationales [En ligne], vol. 22 - n² | 2006, mis en ligne le 27 novembre 2008, consulté le 15 avril 2022. URL : http:// journals.openedition.org/remi/4094; DOI : https://doi.org/10.4000/remi.4094

Ce document a été généré automatiquement le 15 avril 2022.

(c) Université de Poitiers 


\title{
Thomas LACROIX, Les réseaux
} marocains $d u$ développement - géographie du
transnational et politiques du territorial

\author{
Christian Pihet
}

\section{RÉFÉRENCE}

Thomas LACROIX, Les réseaux marocains du développement - géographie du transnational et politiques du territorial, Paris, Les Presses de Sciences Po, 2005, 257 pages (collection Académique), ISBN 2-7246-955-7.

1 L'ouvrage de Thomas Lacroix, adapté d'une thèse de géographie et de sciences politiques soutenue au CERI en 2003, étudie les projets de développement des migrants d'une région du Sud marocain et se plaçant plutôt sur les terrains ruraux, analyse leurs conséquences sociales et politiques pour les villages d'origine. Ce livre ne constitue cependant pas une étude monographique «de plus » mais au contraire, permet à l'auteur d'entamer une réflexion plus ample sur la genèse et le fonctionnement d'un espace transnational entre Europe et Maroc. Pour mener à bien cet objectif, Thomas Lacroix mobilise les concepts et les méthodes des deux sciences sociales précitées. Mobilisation qui aboutit à un travail s'attachant à dégager les effets politiques localisés et inédits d'un triangle formé par les réseaux transnationaux, les pouvoirs publics et les processus de développement.

2 Le livre s'organise en six chapitres regroupés de façon équilibrée en deux grandes parties, la première intitulée "l'espace transnational du développement » et la seconde "les acteurs du développement face aux logiques de pouvoir». Il comprend aussi une indispensable liste des sigles et acronymes ainsi que quatre cartes. Ces dernières placées avant l'introduction auraient gagnées à être intégrées au fil du texte, en regard 
des analyses auxquelles elles font appel. Ainsi lue d'emblée, la carte 3, page 12, demeure a priori énigmatique en raison de l'emploi d'un fond de carte du Maroc obstinément muet, sans explicitation du maillage administratif choisi et de l'utilisation d'acronymes dont on ne va découvrir la signification et surtout l'intérêt qu'au fil de la première partie...

3 L'introduction, solidement charpentée, présente les principaux enjeux du livre, à savoir la définition, le fonctionnement et les effets sociopolitiques d'un espace transnational conçu comme une construction sociale. Les processus de développement territorial sont alors envisagés comme l'un des marqueurs privilégiés de la réalité de cet espace et par conséquence comme l'un des vecteurs du changement social dans le Sud marocain mais aussi chez les émigrés.

4 La première partie, centrée sur les acteurs et les stratégies de construction de cet espace transnational, est fondée sur un solide travail de terrain associant observations, enquêtes et entretiens. Elle présente d'abord la région d'étude, puis les migrantsacteurs et enfin le niveau supérieur de structuration, celui des associations de développement localisées en Europe dans les pays d'accueil de l'émigration. L'espacelaboratoire retenu est celui des Berbères Chleuhs, dans le sud du royaume, polarisé pour partie par Agadir et situé entre l'Atlas et les plaines atlantiques. Dix-sept villages ont été retenus au Maroc, ainsi que cinq associations d'émigrés en France, Belgique et Pays Bas. Des fragments significatifs des entretiens ont été insérés dans le texte, donnant ainsi chair aux raisonnements plus abstraits de l'auteur. L'ensemble de la documentation atteste la grande familiarité de Thomas Lacroix avec cette région, familiarité qui l'aide à structurer sa démonstration à l'aide d'exemples précis. Il en résulte une description détaillée des collectifs d'émigrés Kasbati (province de Tata) et surtout des «leaders du développement » (pages 69-81).

5 En combinant l'étude des projets et des types d'acteurs, Thomas Lacroix joue constamment sur les différentes échelles, du niveau micro-local à l'européen et ce jeu des échelles constitue l'un des points forts de sa démonstration puisqu'elle donne cohérence à ces allers et retours, à «l'ici et au là-bas ", entre les deux rives... On suit avec intérêt, en terrain français principalement, son analyse postérieure des associations, les OSIM (organisations de solidarité internationale issues de la migration) tant celles provenant de la mouvance progressiste marocaine que celles jugées par lui d'influence plus européennes comme par exemple Migrations et Développement. Il résulte de cette bonne centaine de pages un tableau foisonnant des flux entre Maroc et Europe. L'un des intérêts majeurs de ces passages réside dans le croisement des compétences de politiste et de géographe de Thomas Lacroix, à savoir son souci de lier en permanence les niveaux d'intervention politique avec les projets et les organisations locales en recourant pour l'analyse à des jeux d'échelles diversifiées. Faut-il le suivre complètement quand il affirme à la fin du chapitre 3, après avoir décrit l'espace des migrants comme hétérogène et construit sur la fracture entre l'ici et le là-bas, qu'il s'agit d'un espace transnational devenu "une entité sociale autonome» (page 133) ? Le recenseur en doute et nous reviendrons ultérieurement sur cette question.

Néanmoins, il est évident que ses enquêtes fouillées sur les projets de développement dans, par exemple, les villages d'Attacharouk ou de Kasbat (pages 69, 85) représentent une illustration convaincante des modalités du changement social et de l'acculturation au bénéfice des modèles européens. De même, il insiste avec pertinence sur le couple associant projets de développement et constructions des spatialités et sa résultante sur 
l'identité migratoire, en devenir dans cette région, celle, selon lui, du "transmigrant » (pages 92-98).

7 La seconde partie se compose également de trois chapitres examinant l'emboîtement des dispositifs publics d'aide au projet, du village kasbati à l'échelle de la Commission Européenne. Le chapitre 4 se situe clairement dans la logique d'analyse locale de la partie précédente, à savoir une étude centrée sur les rapports entre le développement local et les jeux de pouvoir dans le Sud marocain. On y lit avec intérêt, grâce aux extraits d'entretiens, l'analyse des tensions produites par les projets entre les élites locales et les structures étatiques du Maroc. De fait, l'ouvrage de Thomas Lacroix nous fait assister à l'affirmation et à la diversification d'une société politique locale. Les migrants et leurs projets agissent en tant que révélateurs des conflits portant sur la maitrise et l'aménagement des territoires ruraux, conflits opposant des élites villageoises beaucoup plus réactives que jadis aux pouvoirs centraux et à leurs relais locaux. Cependant à ce stade du développement local, les migrants n'en tirent pas grand bénéfice et tendent à être renvoyés à leurs statuts d'émigrés et replacés dans le champ transnational.

8 Le chapitre qui suit sur les politiques migratoires européennes, texte nettement distancié des terrains du Sud marocain, induit une rupture logique dans l'agencement de l'ouvrage et n'apporte pas grand chose de supplémentaire aux lecteurs avertis. En revanche le dernier chapitre revient sur les acteurs non étatiques et leur place dans le co-développement. Le géographe regrette la distance prise ici encore avec les espaces scrutés dans la première partie car les analyses présentées sont souvent issues d'une revue des ouvrages et rapports existant sur le sujet. Notons cependant l'étude du jumelage entre Figuig et la Seine-Saint-Denis et surtout en fin de chapitre un examen critique du co-développement dont l'auteur dénonce les conceptions un peu trop rigides comme par exemple l'obsession de la maîtrise des flux alors que les enquêtes ne font pas apparaître de liens systématiques entre pauvreté et migration.

Pour conclure, ce travail nous semble utile et pertinent, notamment en raison des informations inédites qu'il fournit sur les interactions entre flux migratoires et les projets de développement en milieu rural et montagnard. Il s'appuie également sur une vaste bibliographie largement pluridisciplinaire.

10 Néanmoins, il apparaît difficile de suivre complètement l'auteur dans sa thèse sur l'espace transnational euro-marocain et les structurations identitaires qu'il produit. Ces processus ont été déjà décrits antérieurement pour d'autres espaces, processus qui à notre avis dépassent le cadre politique du national ou du transnational et sont assez largement recouverts par le concept de « champ migratoire » défini par Gildas Simon.

11 Cependant l'attention vigilante portée aux évolutions identitaires, aux liens entre migrations, projets de développement et tensions politiques nous interroge. À cet égard le Sud marocain des Berbères Chleuhs apparaît comme un véritable laboratoire du développement local où se forgent par strates successives des identités superposées (villageois, migrant, «fransaoui») et où s'affirme progressivement, en lien avec les projets des migrants, un espace politique local. Ce n'est pas le moindre mérite de ce livre riche en matériau et en efforts de conceptualisation que d'attirer notre attention sur ces questions. 


\section{AUTEURS}

\section{CHRISTIAN PIHET}

UMR CNRS 6590, ESO Université d'Angers 